\title{
Weltweit präsenter
}

\section{Liebe Leserin, lieber Leser,}

im Editorial der letzten Ausgabe 2015 haben wir Ihnen mehr Internationalität der Automobilfachtitel für dieses neue Jahr angekündigt - und starten gleich zu Beginn. Im Interview beispielsweise lesen Sie, wo das oft unterschätzte Know-how des US-amerikanischen Autobauers Tesla steckt, der seine Fahrzeuge dank einer ganzheitlichen ITSecurity-Strategie so engmaschig und interaktiv vernetzen kann wie kein anderer OEM heute.

Doch allein internationale Autoren und Themen genügen sicherlich nicht, dem Anspruch der stärkeren weltweiten Präsenz gerecht zu werden. Die Abonnenten unserer Worldwide-Ausgaben von ATZ, MTZ und ATZelektronik konnten bislang nur Fachbeiträge in einer englischen Fassung als PDF-Datei lesen. Nun bieten wir ein komplettes E-Magazin auf Englisch - mit allem, was Sie aus der deutschen Ausgabe kennen: Unsere Rubrik „Im Fokus“, die Titelstrecke mit Interview, Nachrichten über Unternehmen und neue Produkte, der Gastkommentar und natürlich die Fachbeiträge aus Industrie und Forschung lassen sich ab sofort komfortabel auf Englisch und mit neuen Funktionen lesen.

Sie können das internationale elektronische Magazin bequem in jedem modernen Internetbrowser abrufen. Und das unabhängig vom jeweiligen Betriebssystem und davon, ob Sie einen PC, ein Tablet oder ein Smartphone benutzen. Das Herunterladen sowie der Zugriff über eine App ist somit nicht mehr nötig. Der dafür nötige HMTL5-Standard erlaubt es uns auch, künftig Multimedia-Inhalte einzubinden und Ihnen damit echten Mehrwert zu bieten. Mit dem interaktiven Inhaltsverzeichnis springen Sie schnell zu einem Beitrag; mit der Suchfunktion können Sie rasch die gesamte Ausgabe nach einem
Schlagwort durchforsten. Von jeder Seite aus ist es zudem möglich, direkt auf verschiedene Soziale Medien zuzugreifen, um Ihre Kollegen auf interessante ATZelektronik-Inhalte hinzuweisen. Oder aber auch, den Inhalt ganz klassisch als PDF abzuspeichern oder auszudrucken.

Schauen Sie doch einmal rein unter www.atzelektronik-worldwide.com oder empfehlen Sie unsere neuen, englischsprachigen E-Magazine Ihren Kollegen in aller Welt. Unter der Adresse finden Sie übrigens auch den gesamten Jahrgang 2015 als E-Magazin. Und das klassische PDF-Archiv bleibt selbstverständlich erhalten.

Internationaler wird auch die ATZ-Fachtagung „Fahrerassistenz - von der Assistenz zum automatisierten Fahren“ am 13. und 14. April 2016 in Frankfurt am Main. Dort wird übrigens deutlich, dass Kryptografie-Experten aus dem „deutschen Silicon Valley Bochum“, weltweit gefragt sind und beispielsweise in den USA Führungspositionen übernehmen.

Gutes Gelingen in 2016 wünscht Ihnen,

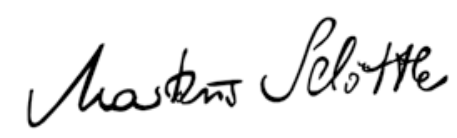

Markus Schöttle

Stellvertretender Chefredakteur

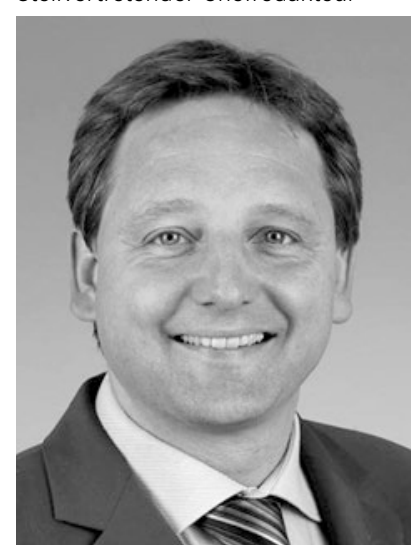

Die neue

Performance

\section{in der optischen \\ Schwingungsmessung}

Noch verlässlicher, noch

vielseitiger, noch besser

ATZ elektronik $0112016 \quad$ 11. Jahrgang 